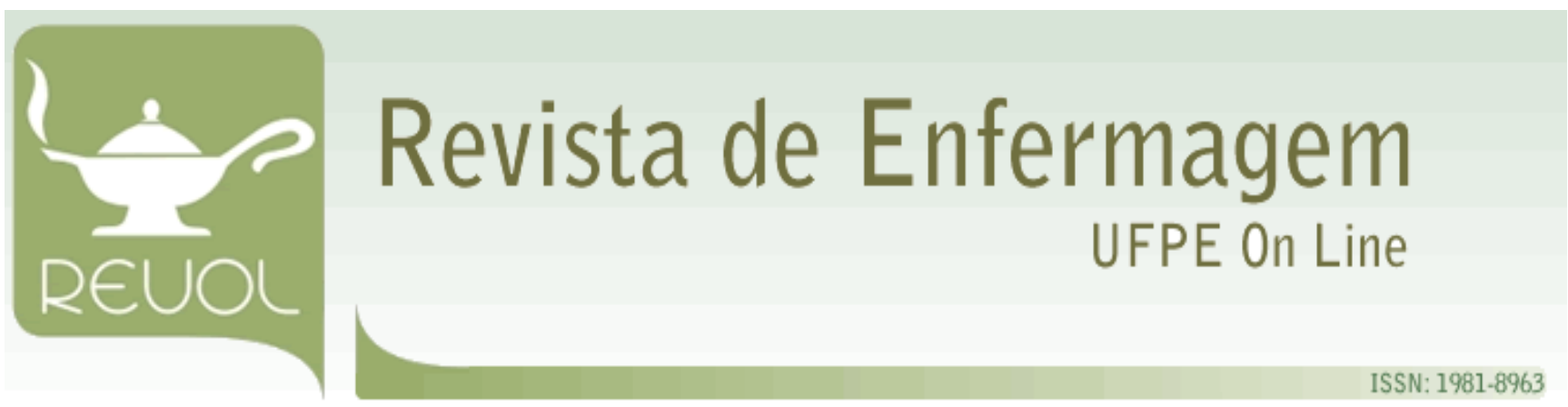

\title{
EDITORIAL
}

Ednaldo Cavalcante de Araújo. Enfermeiro. Professor Doutor do Departamento de Enfermagem da Universidade Federal de Pernambuco - UFPE - Recife (PE), Brasil. Pós-doutorando em Sorbonne, Paris - França (FR). E-mail: ednenjp@gmail.com

\section{THE INDEXING OF JOURNAL OF NURSING UFPE ON LINE - JNUOL}

It is with joy and satisfaction of accomplishment that we are publishing the second volume of REUOL, which has a special meaning because it was the first birthday and we are pleading the indexing in 2008 year. We know that for happening the transfer of information, ie, dissemination of new knowledge, care begins with the drafting of the manuscript, go through the choice of journal for publication and go to the indexing in databases.

Index is understood as the act of describing and identifying the document for its content. Is the basic process in information retrieval. By indexing, determine the subject of a document - object that provides us with information - which is represented according to the descriptors of the documentary language adopted by the librarian.

In turn, be scientific editor is one of the most fascinating and challenging professional activities with which I was involved. It is a privilege to have taken up this task by means of which the JNUOL lends his significant contribution to the growth of publications in the field of nursing, particularly, and in related areas, whether in Brazil or other countries with higher-quality production of knowledge, providing new information results - to other scientists researching the same subject and also to other parties.

However, a key challenge, though, is to increase the visibility of JNUOL, whose response will translate into several indexes on databases that have international and national long for. It is worth noting that we already have some prerequisites, such as:

1. Obedience to the Vancouver Convention;

2. Peer review;

3. Titles and abstracts of all articles in English and Spanish;

\section{Editorial;}

5. Acceptance of papers in English, Spanish and French;

6. Articles written by foreign researchers.

Another achievement of greater importance is the electronic availability through the Scielo (Scientific Electronic Library Online, BIREME), which will guarantee free access, free and democratic to the full text of the Journal on the Internet.

The JNUOL is current in their content and maintaining the regularity of their publications. Improvements were implemented in the normalization of the Journal and the editorial procedures. National and international researchers in various fields of knowledge are part of the Editorial Board.

Because of these improvements have been great interest in the scientific community by JNUOL and we are sure that research and publish articles that are extremely relevant in some subjects and would be cited if it were indexed the Index Medicus and Medline that are still our biggest goals.

However, this trajectory involved and still involves, of course, hard work and personal commitment, but could not be as effective without the support received in the first several years. I thank all those who attended and believed in our dedication and seriousness. I thank those who worked with me, supporting me and prestige - authors, national and international reviewers, editors and layout, secretary and members of the editorial - which greatly contribute to the journal reaches the quality standard features that today.

To all, my sincere thanks!

\section{A INDEXAÇÃO DA REVISTA DE ENFERMAGEM UFPE ON LINE - REUOL}

É com alegria e satisfação do dever cumprido que estamos publicando o volume dois da REUOL, que tem um significado todo 
especial por se tratar do primeiro aniversário e estarmos pleiteando a indexação nesse ano de 2008. Sabemos que, para que aconteça a transferência da informação, isto é, a divulgação de novos conhecimentos, os cuidados se iniciam com a redação do manuscrito, passam pela escolha do periódico para publicação e vão até a indexação em bases de dados.

Entende-se indexar como 0 ato de descrever e identificar o documento pelo seu conteúdo. Constitui o processo básico na recuperação da informação. Por meio da indexação, determina-se o assunto de um documento - objeto que nos fornece informações - o qual é representado de acordo com os descritores da linguagem documentária adotada pelo bibliotecário.

Por sua vez, ser editor científico é uma das mais fascinantes e desafiadoras das atividades profissionais com as quais me envolvi. É um privilégio ter assumido esta tarefa por meio da qual a REUOL presta sua significativa contribuição para o crescimento das publicações na área da Enfermagem, principalmente, e nas áreas afins, seja do Brasil ou de outros países com aumento da produção qualificada do conhecimento, fornecendo novas informações - resultados - a outros cientistas que pesquisam o mesmo assunto e também a outros interessados.

Todavia, um desafio fundamental, ainda, é aumentar a visibilidade da REUOL, cuja resposta se traduzirá em várias indexações em bases de dados internacionais e nacionais que ainda almejamos. Vale notar que já temos alguns pré-requisitos, tais como:

1. Obediência à Convenção de Vancouver;

2. Revisão por pareceristas;

3. Títulos e resumos de todos os artigos em inglês e espanhol;

4. Editorial;

5. Aceitação de artigos em inglês, espanhol e francês;

6. Artigos escritos por pesquisadores estrangeiros.

Outra conquista da maior relevância será a disponibilização eletrônica, por meio do Scielo (Scientific Electronic Library Online, Bireme), que garantirá acesso livre, democrático e gratuito aos textos completos da Revista pela Internet.

A REUOL está atualizada em seu conteúdo e mantendo a regularidade de suas publicações. Foram implementadas melhorias na normalização da Revista e nos procedimentos editoriais. Pesquisadores nacionais e internacionais de várias áreas do conhecimento integram a Equipe Editorial.

Devido a estes melhoramentos tem havido grande interesse da comunidade científica pela REUOL e temos a certeza de que as pesquisas e os artigos que publicamos são extremamente relevantes em alguns temas e seriam mais citados se fossem nele indexados o Index Medicus e o Medline que são ainda nossos maiores objetivos.

No entanto, esta trajetória envolveu e ainda envolve, certamente, muito trabalho e comprometimento pessoal, mas não poderia ser tão eficaz sem os vários apoios recebidos neste primeiro ano. Agradeço a todos aqueles que nos prestigiaram e que acreditaram na nossa dedicação e seriedade. Agradeço aos que comigo trabalharam, prestigiam-me e apóiam - autores, revisores nacionais e internacionais, editores de texto e de layout, secretária e membros do Conselho Editorial que em muito contribuem para que a Revista alcance o padrão de qualidade que hoje apresenta.

A todos, meus sinceros agradecimentos!

\section{LA INDEXACIÓN DE LA REVISTA DE ENFERMERÍA UFPE ON LINE - REUOL}

Es con alegría y satisfacción de logro que estamos publicando el segundo volumen de REUOL, que tiene un significado especial porque es el primer cumpleaños y buscaremos la indexación en año 2008. Sabemos que eso suceda para la transferencia de información, es decir, la difusión de nuevos conocimientos, la atención se inicia con la redacción del manuscrito, pasar por la elección de la revista para su publicación e ir a la indexación en bases de datos. Indexar se entiende como el acto de describir e identificar el documento por su contenido. Es el proceso básico de recuperación de información.

Por indexación, determina el objeto de un documento - objeto que nos proporciona la información - que se representa de acuerdo a los descriptores del lenguaje documental adoptado por el bibliotecario.

A su vez, ser editor científico es una de las actividades profesionales más fascinantes y difíciles con los que estuve involucrado. Es un privilegio haber asumido esta tarea por medio del cual el REUOL presta su contribución significativa al crecimiento de las publicaciones en el campo de la enfermería, en particular, y en áreas relacionadas, ya sea en Brasil o en otros países con una producción de mayor calidad del conocimiento, proporcionando nueva información - 
resultados - a otros cientistas investigando el mismo tema y también a otras partes.

Sin embargo, un desafío clave, es aumentar la visibilidad de REUOL, cuya respuesta se traducirá en varios bases de datos que han nacionales e internacionales. Cabe destacar que ya tenemos una serie de condiciones, tales como:

1. La obediencia a la Convención de Vancouver;

\section{Revisión por pares;}

3. Los títulos y resúmenes de todos los artículos en Inglés y Español;

4. Editorial;

5. La aceptación de documentos en Inglés, español y francés;

6. Artículos escritos por investigadores extranjeros.

Otro de los logros de mayor importancia es la disponibilidad electrónica a través de la colección SciELO (Scientific Electronic Library Online, BIREME), que se garantice el libre acceso, libre y democrático con el texto completo de la revista en Internet.

El REUOL es actual en su contenido y mantener la regularidad de sus publicaciones. Las mejoras se llevaron a cabo en la normalización de la Revista y los procedimientos editoriales. Investigadores nacional e internacional en diversas áreas del conocimiento forman parte del Consejo Editorial.

Debido a estas mejoras han sido un gran interés en la comunidad científica por REUOL y estamos seguros de que la investigación y publicar artículos que son muy relevantes en algunos temas y se mencione si se indexan el Index Medicus y Medline que aún nuestros mayores metas.

Sin embargo, en este trayecto y aún así implica, por supuesto, el trabajo duro y compromiso personal, pero no podría ser tan efectiva sin el apoyo recibido en los primeros años. Agradezco a todos los que asistieron y creyeron en nuestra dedicación y seriedad. Doy las gracias a los que han trabajado conmigo, que me apoya y prestigio - autores, revisores nacionales e internacionales, los editores y el secretario de diseño, y los miembros de la redacción - que contribuyen en gran medida a la revista llega a las características de calidad estándar que hoy en día.

A todos, mi más sincero agradecimiento! 